\title{
Subsurface water fluxes and their controls in a sloping heterogeneously layered volcanic soil beneath a supra-wet tropical montane cloud forest (NW Costa Rica)
}

\author{
Tobon Conrado ${ }^{1}$ and L. Adrian Bruijnzeel ${ }^{2}$ \\ ${ }^{1}$ Universidad Nacional de Colombia Sede Medellin \\ ${ }^{2}$ Vrije Universiteit Amsterdam Faculteit der Exacte Wetenschappen
}

June 1, 2021

\begin{abstract}
Tropical montane cloud forests (TMCF) receive additional ('occult') inputs of water from fog and wind-driven rain. Together with the concomitant reduction in evaporative losses, this typically leads to high soil moisture levels (often approaching saturation) that are likely to promote rapid subsurface flow via macropores. Although TMCF make up an estimated $6.6 \%$ of all remaining montane tropical forest and occur mostly in steep headwater areas that are protected in the expectation of reduced downstream flooding, TMCF hillslope hydrological functioning has rarely been studied. To better understand the hydrology of a TMCF on heterogeneously layered volcanic ash soils under very high net rainfall conditions (up to 6,740 mm yr-1), we examined temporal and spatial soil water dynamics and their contribution to subsurface runoff and stormflow for a year (1 July 2003 - 30 June 2004) in a small headwater catchment on the Atlantic (windward) slope near Monteverde, NW Costa Rica. Particular attention was paid to partitioning of water fluxes into lateral subsurface flow and vertical percolation. The presence of a gravelly volcanic layer at shallow depth of very high hydraulic conductivity intercalated between two layers of lower conductivity, controlled both surface infiltration and delayed vertical water movement deeper into the soil profile. Soil water fluxes during rainfall were dominated by rapid lateral flow in the gravelly layer, particularly at high soil water contents. In turn, this lateral subsurface flow controlled the magnitude and timing of stormflow from the catchment. Stormflow amount increased rapidly once topsoil moisture exceeded a threshold value of $\sim 0.58 \mathrm{~cm} 3 \mathrm{~cm}-3$. Responses were not affected appreciably by rainfall intensity because soil hydraulic conductivities across the profile largely exceeded prevailing rainfall intensities.
\end{abstract}

\section{Hosted file}

Tobon and Bruijnzeel HYP.docx available at https://authorea.com/users/417202/articles/524386subsurface-water-fluxes-and-their-controls-in-a-sloping-heterogeneously-layeredvolcanic-soil-beneath-a-supra-wet-tropical-montane-cloud-forest-nw-costa-rica 


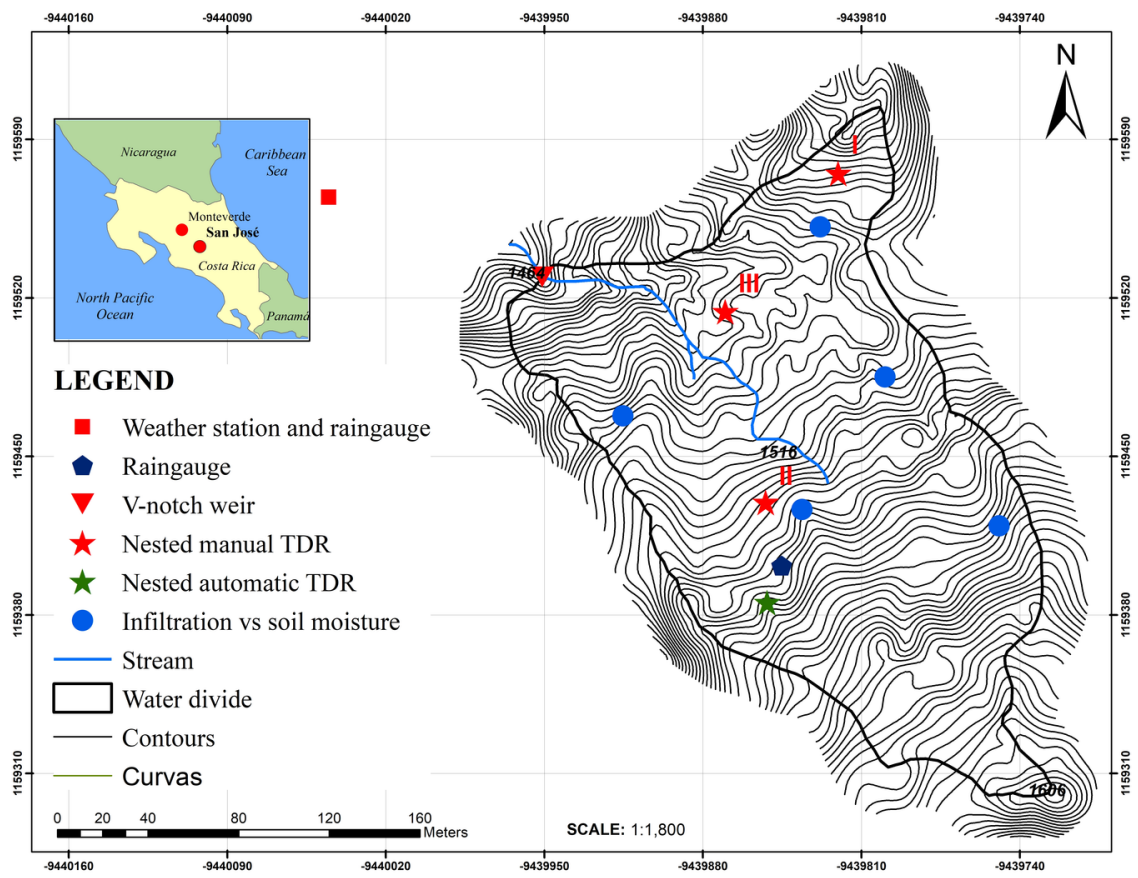

\section{Hosted file}

Figure 2.docx available at https://authorea.com/users/417202/articles/524386-subsurfacewater-fluxes-and-their-controls-in-a-sloping-heterogeneously-layered-volcanic-soilbeneath-a-supra-wet-tropical-montane-cloud-forest-nw-costa-rica

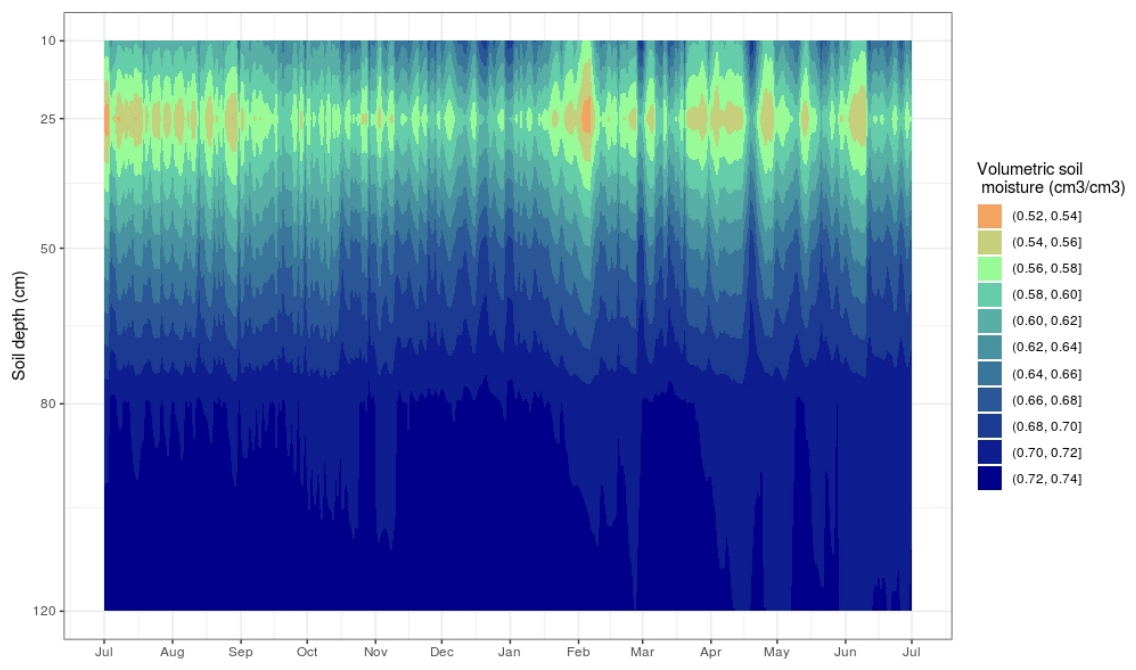




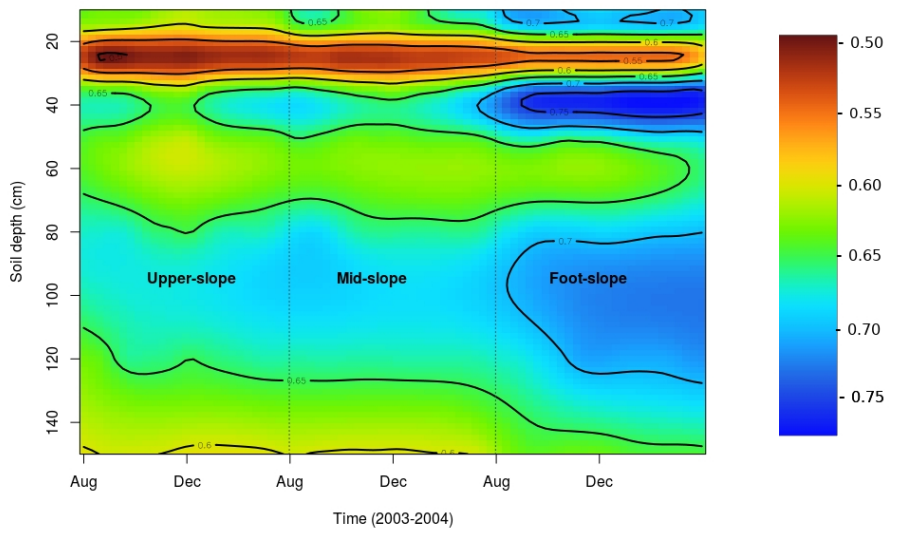

\section{Hosted file}

Figure 5.docx available at https://authorea.com/users/417202/articles/524386-subsurfacewater-fluxes-and-their-controls-in-a-sloping-heterogeneously-layered-volcanic-soilbeneath-a-supra-wet-tropical-montane-cloud-forest-nw-costa-rica

\section{Hosted file}

Figure 6.docx available at https://authorea.com/users/417202/articles/524386-subsurfacewater-fluxes-and-their-controls-in-a-sloping-heterogeneously-layered-volcanic-soilbeneath-a-supra-wet-tropical-montane-cloud-forest-nw-costa-rica

\section{Hosted file}

Figure 7.docx available at https://authorea.com/users/417202/articles/524386-subsurfacewater-fluxes-and-their-controls-in-a-sloping-heterogeneously-layered-volcanic-soilbeneath-a-supra-wet-tropical-montane-cloud-forest-nw-costa-rica

\section{Hosted file}

Table 1.docx available at https://authorea.com/users/417202/articles/524386-subsurface-waterfluxes-and-their-controls-in-a-sloping-heterogeneously-layered-volcanic-soil-beneath-asupra-wet-tropical-montane-cloud-forest-nw-costa-rica

\section{Hosted file}

Table 2.docx available at https://authorea.com/users/417202/articles/524386-subsurface-waterfluxes-and-their-controls-in-a-sloping-heterogeneously-layered-volcanic-soil-beneath-asupra-wet-tropical-montane-cloud-forest-nw-costa-rica

\section{Hosted file}

Table 3.docx available at https://authorea.com/users/417202/articles/524386-subsurface-waterfluxes-and-their-controls-in-a-sloping-heterogeneously-layered-volcanic-soil-beneath-asupra-wet-tropical-montane-cloud-forest-nw-costa-rica

\section{Hosted file}

Table 4.docx available at https://authorea.com/users/417202/articles/524386-subsurface-waterfluxes-and-their-controls-in-a-sloping-heterogeneously-layered-volcanic-soil-beneath-a- 
supra-wet-tropical-montane-cloud-forest-nw-costa-rica 